\title{
Optical properties of peroxy radicals in silica: Multiconfigurational perturbation theory calculations
}

\author{
Carmen Sousa ${ }^{a}$ and Coen de Graaf \\ Departament de Química Física i Centre de Recerca en Química Teòrica, Universitat de Barcelona, Martí $i$ \\ Franquès 1, E-08028 Barcelona, Spain \\ Gianfranco Pacchioni \\ Dipartimento di Scienza dei Materiali, Università di Milano-Bicocca, Istituto Nazionale per la Fisica della \\ Materia, via R. Cozzi 53-I-20125 Milano, Italy
}

(Received 28 November 2000; accepted 26 January 2001)

\begin{abstract}
The low-lying electronic transitions in a peroxy radical, $\equiv \mathrm{Si}-\mathrm{O}-\mathrm{O}^{*}$, a fundamental radiation induced point defect in silicon dioxide, have been investigated by means of ab initio multiconfiguration perturbation theory calculations, CASPT2, and cluster models. The accuracy of the computed transition energies and intensities as predicted by the CASPT2 approach has been checked by studying the exited state properties of two molecular analogues, the $\mathrm{HOO}^{\circ}$ and $\mathrm{CH}_{3} \mathrm{OO}^{\circ}$ radicals, and of a well-characterized paramagnetic defect in silica, the nonbridging oxygen center, $\equiv \mathrm{Si}-\mathrm{O}^{\circ}$, for which unambiguous assignments exist. We found that the peroxy radical gives rise to two optical absorption bands, a very weak one at $0.7 \mathrm{eV}$ and an intense one at $5.49 \mathrm{eV}$, in agreement with the experimental assignments of Radzig [V. A. Radzig, Chem. Phys. Reports 14, 1206 (1995)]. (C) 2001 American Institute of Physics. [DOI: 10.1063/1.1355986]
\end{abstract}

\section{INTRODUCTION}

Point defects in $\mathrm{SiO}_{2}$ play a fundamental role in determining the properties and characteristics of microelectronic and optoelectronic devices in technological applications. ${ }^{1,2}$ Their identification and structural characterization is based on two major spectroscopic techniques, optical absorption (OA) and photoluminescence (PL) spectra and, for paramagnetic defects, electron paramagnetic resonance (EPR). Thanks to the combined use of the these spectroscopies, it has been possible to identify a series of spectral features in pure and doped silica and to assign them to a given structural defect. ${ }^{1,2}$ However, several of these assignments are still controversial and are not universally accepted. A significant improvement in recent years has been represented by the possibility to compute from first principles the optical and magnetic properties of a series of diamagnetic and paramagnetic defects. ${ }^{3-14}$ In particular, the possibility to compute excited state properties, absorption and emission energies, and the relative intensities has permitted us to shed some light into the complex spectral behavior of several point defects in silica. ${ }^{15}$

One of the defects which has attracted considerable experimental attention is the peroxy radical. ${ }^{16}$ Peroxy radicals (POR: $\equiv \mathrm{Si}-\mathrm{O}-\mathrm{O}^{\circ}$ ) are among the most fundamental radiation induced defects in amorphous silicon dioxide together with the $\mathrm{E}^{\prime}$ center $\left(\equiv \mathrm{Si}^{\circ}\right),{ }^{17}$ the nonbridging oxygen hole center $\left(\mathrm{NBO}: \equiv \mathrm{Si}-\mathrm{O}^{\circ}\right)^{18}$ and the self-trapped holes $(\mathrm{STH}$ : $\left.\equiv \mathrm{Si}-\mathrm{O}^{\circ}-\mathrm{Si} \equiv\right) .{ }^{19}$ The POR radical exhibits a surprising behavior since the concentration of this defect grows with annealing temperature and radiation dose, while the concentrations of NBO and $\mathrm{E}^{\prime}$ centers decrease. It has been

${ }^{a)}$ Corresponding author: c.sousa@qf.ub.es suggested ${ }^{16,18,20}$ that precursors of the POR centers are the peroxy linkages, $\equiv \mathrm{Si}-\mathrm{O}-\mathrm{O}-\mathrm{Si} \equiv$ :

$$
\equiv \mathrm{Si}-\mathrm{O}-\mathrm{O}-\mathrm{Si} \equiv \rightarrow \equiv \mathrm{Si}-\mathrm{O}-\mathrm{O}^{\bullet}+{ }^{\bullet} \mathrm{Si} \equiv .
$$

This model has been supported by pioneering quantumchemical calculations by Edwards and Fowler. ${ }^{21}$ On the surface of reactive silica POR centers are formed, by reaction of the $\mathrm{E}^{\prime}$ centers with molecular oxygen, ${ }^{22,23}$

$$
\equiv \mathrm{Si}^{\bullet}+\mathrm{O}_{2} \rightarrow \equiv \mathrm{Si}-\mathrm{O}-\mathrm{O}^{\circ} .
$$

The reaction proceeds at $77 \mathrm{~K}$ with almost no activation barrier. NBO and POR centers can coexist and their optical properties have been the subject of an intense debate. NBO has a well-characterized OA band of low intensity around $600 \mathrm{~nm}(2 \mathrm{eV})$ with an oscillator strength of about $10^{-4} ; 24,25$ the absorption at $2 \mathrm{eV}$ gives rise to a PL band at $1.9 \mathrm{eV}^{26,27}$ A second band near $260 \mathrm{~nm}(4.8 \mathrm{eV})$ has also been assigned to NBO. ${ }^{25-30}$ The POR radical, on the contrary, has been proposed as the origin of various bands, from $2 \mathrm{eV}$ up to 7.6 $\mathrm{eV}$. It has been suggested that one band at $5.3-5.4 \mathrm{eV}$ with oscillator strength $f=0.06 \pm 0.02$ is due to POR centers located on the surface of the material, ${ }^{23,31}$ POR has been considered responsible for a band at $7.6 \mathrm{eV}$ with $f=0.65$ $( \pm 50 \%),{ }^{16,18}$ and for a band around $4.8-5 \mathrm{eV}$ nearly coincident with that of NBO. ${ }^{28,30,32}$ Recently, in a carefully combined EPR and optical absorption study of POR centers in gamma-irradiated fused silica, Griscom and Mizuguchi proposed that POR radicals give rise to a transition at 1.97 $\pm 0.01 \mathrm{eV}$ and $f=0.00057 \pm 0.00015,{ }^{33}$ therefore, this band would completely superpose with that of an NBO center which, according to the same study, originates two bands at 2.19 and $2.08 \mathrm{eV}$ with an oscillator strength practically iden- 
tical to that of POR. While the number of proposed assignments for OA of a POR center is quite large, much less is known about the PL properties.

A few calculations have been dedicated to this center, usually dealing with ground state properties. Using a tightbinding approach, O'Reilly and Robertson estimated an absorption for this center at about $5-6 \mathrm{eV}^{34}$ Quantumchemical calculations based on unrestricted M $\phi$ ller-Plesset second-order perturbation theory (UMP2) have shown the existence of a state at $0.5 \mathrm{eV}$ above the ground state corresponding to the electronic transition of the lone pair of the terminal $\mathrm{O}$ atom to the singly occupied $2 p$ orbital on the same atom, ${ }^{35}$ higher transitions were not searched. In a recent $a b$ initio configuration interaction study, ${ }^{5}$ it was suggested that the POR center gives a very weak absorption at $0.1-0.3 \mathrm{eV}$ above the ground state, with a second state around $6.4-6.6 \mathrm{eV},{ }^{5}$ however, the rather small size of the basis sets used and the limited inclusion of correlation effects in these calculations ${ }^{5}$ could easily result in an overestimate of the excitation energy. Clearly, the number of theoretical studies on the excited state properties of the POR radical is scarce and the results are insufficient to corroborate the proposed assignments.

In this work we report the results of state-of-the-art $a b$ initio calculations on the ground and excited states of a POR center in silica. The defect center has been described by cluster models. In order to validate the computational approach, we have also considered the $\mathrm{HOO}^{\circ}$ and the $\mathrm{CH}_{3} \mathrm{OO}^{\circ}$ radical molecules, for which experimental and theoretical data on the excitation energies are available, and models of a NBO center in silica, since this is a well-characterized paramagnetic defect with unambiguous assignments of the optical transitions. The stability of the results versus basis sets, extension of electron correlation effects, and method adopted for the optimization of the ground state geometry (UMP2 or density functional theory) have been carefully checked. The results provide compelling evidence that the POR center gives rise to an absorption band around $5.5 \mathrm{eV}$ and that no other transitions exist below this threshold, except for a very low intensity band at $0.7 \mathrm{eV}$.

\section{COMPUTATIONAL DETAILS}

The POR center has been modeled by an $(\mathrm{OH})_{3} \mathrm{SiOO}$. cluster and for the $\mathrm{NBO}$ center we use an $(\mathrm{OH})_{3} \mathrm{SiO}$. cluster model. These models have proven to be sufficient to study the spectroscopy of point defects in silica because of the very local nature of the transitions. ${ }^{5}$ The cluster size dependence of the results has been carefully checked by Stefanov and Raghavachari ${ }^{11}$ and Raghavachari and Pacchioni ${ }^{36}$ for some specific point defects in silica. It has been shown that, with respect to the clusters used here, the inclusion of the next shell of Si atoms affects the excitation energies by about $0.2-0.3 \mathrm{eV}$, while the inclusion of another shell of $\mathrm{O}$ atoms has no effect on the transitions. ${ }^{11,36}$

For all four systems studied here, ground state geometries have been optimized with the B3LYP hybrid density functional or by UMP2 applying the augmented correlation consistent polarized valence double-zeta (aug-cc-pVDZ) basis $\operatorname{set}^{37}$ for all atoms, i.e., a $[5 s, 4 p, 2 d]$ contraction for $\mathrm{Si}$,

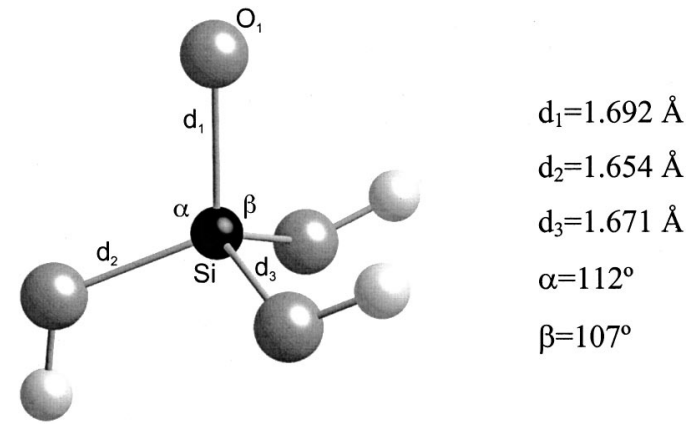

FIG. 1. B3LYP optimized structural parameters of the ground state of the $(\mathrm{OH})_{3} \mathrm{SiO}$. cluster to model the NBO defect in silica.

$[4 s, 3 p, 2 d]$ for $\mathrm{C}$ and $\mathrm{O}$, and $[3 s, 2 p]$ for $\mathrm{H}$. No symmetry restrictions have been imposed on the optimization procedure. Nevertheless, in all systems the optimized structures transform following the $C_{s}$ point group symmetry. Figures 1 and 2 show the most relevant geometrical parameters obtained in the B3LYP optimization of the NBO and POR structures. UMP2 closely reproduces these parameters, as the differences in the distances are smaller than $0.02 \AA$ in all cases and angles do not differ by more than $2 \mathrm{deg}$. The influence on the transition energies will be discussed in the next section. It is worth mentioning that this behavior is not universal and that there are cases, like, for instance, that of $\mathrm{Si}=\mathrm{O}$ double bonds, where the MP2 and B3LYP optimizations give quite different results with non-negligible effects on the transition energies. ${ }^{36}$

The computational approach employed to construct accurate $\mathrm{N}$-electron wave functions for ground and excited states is the complete-active-space second-order perturbation theory (CASPT2) developed by Anderson and coworkers. ${ }^{38,39}$ CASPT2 is a generalization of the well-known MP2 method based on Hartree-Fock wave functions to complete-active-space SCF (CASSCF) wave functions. An important part of the electron correlation effects is treated in a variational way in the CASSCF step, and the remainder, mainly dynamical electron correlation, is estimated by second-order perturbation theory with the CASSCF wave function as a zeroth-order wave function. This strategy combines the accuracy of a multi-reference CI treatment and the low computational cost of a perturbational approach. Over the last few years the CASPT2 method has proven to be a fruitful approach to study, analyze, and predict the spectros-

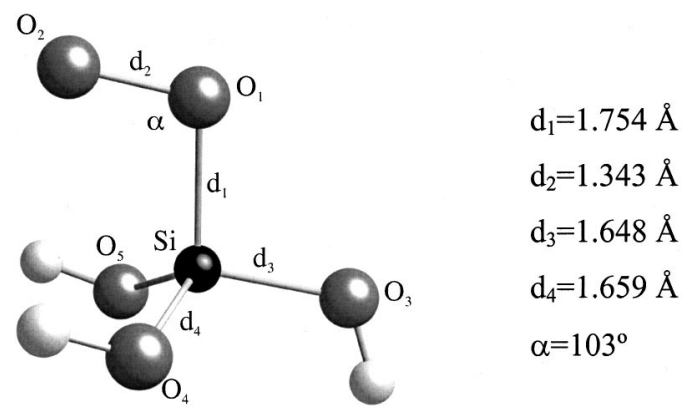

FIG. 2. B3LYP optimized structural parameters of the ground state of the $(\mathrm{OH})_{3} \mathrm{SiOO}$. cluster to model the POR defect in silica. 
copy of a wide range of organic and inorganic molecules. $^{40-43}$ The method has also been successfully applied to study excited states in solid state compounds. ${ }^{44-46}$

The construction of the CASSCF wave functions has been performed in the $C_{s}$ point group symmetry. To avoid convergence problems and to ensure orthogonal ground and excited states, the CASSCF wave functions have been optimized for an average of states for each irreducible representation of the $C_{s}$ point group symmetry, $A^{\prime}$ and $A^{\prime \prime}$. Large active spaces have been used that include all important valence orbitals augmented with a set of virtual orbitals. In this way, a large part of the electron correlation effects is treated variationally. In the subsequent CASPT2 step, a secondorder estimate of the total energy is calculated for each electronic state separately by correlating all valence electrons, i.e., $\mathrm{Si}-3 s, 3 p, \mathrm{C}-2 s, 2 p, \mathrm{O}-2 s, 2 p$, and $\mathrm{H}-1 s$. The oscillator strengths are computed following the standard procedure of the CASSCF/CASPT2 methodology, namely, making use of the transition dipole moments derived from the CASSCF wave functions ${ }^{47}$ and the CASPT2 excitation energies. All excitation energies are obtained as vertical transitions from the equilibrium ground state geometry. Except as stated otherwise, CASSCF/CASPT2 calculations were performed at the B3LYP optimized geometry with the same basis set as in the optimization procedure, i.e., an aug-cc-pVDZ basis set for all atoms. Geometry optimizations have been done with the GAUSSIAN 98 suite of programs. ${ }^{48}$ For the calculations of the spectroscopic parameters we have used the MOLCAS4 package. $^{49}$

\section{PERFORMANCE OF THE COMPUTATIONAL APPROACH: $\mathrm{HOO}$. AND $\mathrm{CH}_{3} \mathrm{OO} \cdot \mathrm{RADICALS}$}

The reliability of the CASSCF/CASPT2 approach to calculate accurate excitation energies for radical species has been checked by applying the methodology to two test cases: the $\mathrm{HOO}$ - and $\mathrm{CH}_{3} \mathrm{OO}$ radicals because of their similarity with the POR defect. The spectroscopy of the HOO- radical is well resolved, both experimentally and theoretically. ${ }^{50-52}$ Basically, two OA peaks are found: a weak transition around $1 \mathrm{eV}$ and a more intense one in the 5.9-6.05 eV range. ${ }^{50-52}$ These transitions have been assigned to excitations from the $a^{2} A^{\prime \prime}$ ground state to the $a^{2} A^{\prime}$ and $b^{2} A^{\prime \prime}$ excited states, respectively. For $\mathrm{CH}_{3} \mathrm{OO}$. the situation is somewhat less clear. A broad intense OA band is observed in the 4.1-5.6 $\mathrm{eV}$ range with a maximum at $5.4 \mathrm{eV}$ (Refs. 53,54, and references therein). A weak peak in the near-infrared region has also been reported at $0.9 \mathrm{eV} .{ }^{55}$ Only a few theoretical calculations on the excited states of this molecule have been published. Jafri and Phillips ${ }^{56}$ studied the potential energy curves of various electronic states with selected CI wave functions. While they rather accurately reproduced the $0.9 \mathrm{eV}$ peak, the $a^{2} A^{\prime \prime} \rightarrow b^{2} A^{\prime \prime}$ transition at $5.4 \mathrm{eV}$ is off by approximately 1 eV. A more recent study of Fahr and co-workers ${ }^{54}$ reported an excitation energy around $0.8 \mathrm{eV}$ for the $a^{2} A^{\prime}$ state and a second transition between 5.4 and $6.0 \mathrm{eV}$ depending on the computational scheme (Hartree-Fock, MP2, or multiconfigurational SCF) followed to optimize the geometry.

In the following, we check the dependence of the calculated excitation energies on the size of the active space, the
TABLE I. CASPT2 excitation energies (in eV) of the $\mathrm{HOO} \cdot$ and $\mathrm{CH}_{3} \mathrm{OO}$. radicals obtained with $\mathrm{CASSCF}$ reference wave functions constructed from active spaces of different size. The small active space only includes the orbitals involved in the transitions. The medium CAS contains a set of valence occupied and virtual orbitals selected by RASSCF calculations. The large CAS extends the previous space with additional virtual orbitals (see text). Computed values are compared with experimental data.

\begin{tabular}{lccccc}
\hline \hline \multirow{2}{*}{ CAS } & \multicolumn{2}{c}{ HOO. } & & \multicolumn{2}{c}{$\mathrm{CH}_{3} \mathrm{OO}}$. \\
\cline { 2 - 3 } \cline { 5 - 6 } \cline { 5 - 6 } & $a^{2} A^{\prime}$ & $b^{2} A^{\prime \prime}$ & & $a^{2} A^{\prime}$ & $b^{2} A^{\prime \prime}$ \\
\hline Small & 1.34 & 6.00 & & 1.45 & 5.34 \\
Medium & 1.08 & 6.16 & & 1.11 & 5.59 \\
Large & 1.08 & 6.16 & & 1.12 & 5.40 \\
Exp. (Refs. 50-54) & 1 & $5.9-6.05$ & & - & 5.4 \\
\hline \hline
\end{tabular}

basis set, and the geometry. Table I reviews the CASPT2 excitation energies for the experimentally observed bands in $\mathrm{HOO}$ - and $\mathrm{CH}_{3} \mathrm{OO}$. computed with three active spaces of different size. The small active space contains only the orbitals involved in the transitions, that is, two orbitals and three electrons for the states of ${ }^{2} A^{\prime}$ symmetry and similarly for those of ${ }^{2} A^{\prime \prime}$ symmetry. A complete valence active space becomes rather large, especially for the $\mathrm{CH}_{3} \mathrm{OO}$ - radical. Hence, to construct the medium active space, we have performed a restricted-active-space SCF (RASSCF) calculation and included in the active space those orbitals for which the natural orbital occupation numbers show the largest deviation from 2 or 0 . This procedure leads to an active space of 7 orbitals and 9 electrons for HOO-, and 10 orbitals and 13 electrons for $\mathrm{CH}_{3} \mathrm{OO}$. Finally, we extend this active space with three extra virtual orbitals. This active space is referred to as large CAS. As can be readily seen from Table I the excitation energies undergo a significant change going from the small to the medium active space, but the addition of the extra set of virtuals does not significantly affect the relative energies, indicating that the correlation effects due to these orbitals are already treated correctly by perturbation theory with the medium CAS wave function as reference. Comparison with experiment is rather satisfactory; the CASSCF/ CASPT2 method reproduces the transition energies within $\sim 0.1 \mathrm{eV}$, an accuracy found before in many other applications of the method. ${ }^{40}$ For both systems, the calculated oscillator strength of the $a^{2} A^{\prime \prime} \rightarrow b^{2} A^{\prime \prime}$ transition indicates that this transition carries the main part of the spectral weight, while the lowest transition is only very weak as observed experimentally.

We have also studied the basis set dependence of the CASSCF/CASPT2 transition energies. Beside the already mentioned aug-cc-pVDZ basis (see Table I), we have considered two different atomic natural orbital (ANO) contractions from a large primitive set of gaussian type functions. The $(14 s, 9 p, 4 d, 3 f)$ set for $\mathrm{C}$ and $\mathrm{O}$ has been contracted to $[4 s, 3 p, 2 d]$ and $[5 s, 4 p, 3 d, 2 f]$, and the $(8 s, 4 p, 3 d)$ set for $\mathrm{H}$ has been contracted to $[3 s, 2 p]$ and $[3 s, 2 p, 1 d] .{ }^{57}$ With the medium active space defined above, we find that the excitation energies computed with both ANO basis sets are slightly lower than the values reported in Table I, but the largest deviation is less than $0.08 \mathrm{eV}$. Hence, it can be safely concluded that the aug-cc-pVDZ basis set is large enough to 
TABLE II. CASPT2 excitation energies $T_{e}$ (in $\mathrm{eV}$ ) and oscillator strengths $f$ of the $(\mathrm{OH})_{3} \mathrm{SiO}$. radical to model the nonbridging oxygen defect in $\mathrm{SiO}_{2}$. The active space contains 13 orbitals and 13 electrons. CASPT2 leaves the core electrons ( $\mathrm{Si}-1 s, 2 s, 2 p$, and $\mathrm{O}-1 s)$ frozen. The last two columns list the experimentally observed transition energies and the oscillator strengths.

\begin{tabular}{lccccc}
\hline \hline & \multicolumn{2}{c}{ CASPT2 } & & \multicolumn{2}{c}{ Experiment } \\
\cline { 2 - 3 } \cline { 5 - 6 } State & $T_{e}$ & $f$ & & $T_{e}$ & $f$ \\
\hline$a^{2} A^{\prime \prime}$ & 0.00 & - & & 0.00 & - \\
$a^{2} A^{\prime}$ & 0.24 & $7.7 \times 10^{-6}$ & & - & - \\
$b^{2} A^{\prime}$ & 2.02 & $5.6 \times 10^{-4}$ & & 2.0 (Ref. 25) & $4 \times 10^{-4}$ (Ref. 25) \\
$b^{2} A^{\prime \prime}$ & 4.08 & $5.2 \times 10^{-2}$ & & - & - \\
$c^{2} A^{\prime}$ & 5.02 & $3.5 \times 10^{-2}$ & & 4.8 (Ref. 28) & $2 \times 10^{-1}$ (Ref. 28) \\
\hline \hline
\end{tabular}

study the spectroscopy of this type of radicals. Finally, we have checked the influence of the geometry by comparing the excitation energies calculated for the B3LYP and UMP2 optimized structures for the ground state of both radicals. For $\mathrm{HOO}$ both geometries are very similar, while somewhat more pronounced differences are found for $\mathrm{CH}_{3} \mathrm{OO} \cdot$. In the latter radical, the $\mathrm{O}-\mathrm{O}$ bond length is $0.014 \AA$ shorter in the UMP2 structure and the $\mathrm{C}-\mathrm{O}$ distance is $0.011 \AA$ longer. However, these differences result in very modest changes in the excitation energies; the $a^{2} A^{\prime \prime} \rightarrow b^{2} A^{\prime \prime}$ transition becomes, at most, $0.1 \mathrm{eV}$ higher with the UMP2 geometry, whereas the change in the lowest transition energy does not exceed $0.03 \mathrm{eV}$.

\section{THE NBO CENTER}

The ground state and the low-lying excited states of the NBO defect of silica have been studied using a $(\mathrm{OH})_{3} \mathrm{Si}-\mathrm{O}$. cluster (see Fig. 1). CASSCF/CASPT2 transition energies $\left(T_{e}\right)$ and oscillator strengths $(f)$ are reported in Table II and are compared to the available experimental data. The dependency of $T_{e}$ on the size of the active space is very similar to that observed for the $\mathrm{HOO}$. and $\mathrm{CH}_{3} \mathrm{OO}$. radical molecules reported in the previous section. In other words, the relative energies do not change once a medium-sized active space is used. Increasing the basis set or taking the UMP2 optimized geometry has a negligible effect, and only slightly affects (by less than $0.1 \mathrm{eV}$ ) the $T_{e}$ for the higher excitations. Table II reviews the results for the largest active space considered, which contains the highest occupied valence orbitals and some virtual orbitals. The CASSCF wave functions are constructed by distributing 13 electrons over 13 orbitals in all possible ways.

Although the peaks at $2.0 \mathrm{eV}$ and around $4.8 \mathrm{eV}$ have been unambiguously assigned to the NBO center, the character of the transitions is still unclear. In the following, we discuss the CASPT2 results listed in Table II, compare them with experimental data, and propose an interpretation of the character of the $2.0 \mathrm{eV}$ and $4.8 \mathrm{eV}$ bands attributed to the NBO center. The lowest transition corresponds to a switch of the unpaired electron from the $\mathrm{O}-2 p_{y}$ to $\mathrm{O}-2 p_{x}$ nonbonding orbitals centered on the nonbridging oxygen. In agreement with previous theoretical studies, ${ }^{5}$ CASPT2 predicts the two states to be almost degenerate, $T_{e}$ is $0.24 \mathrm{eV}$, and the very low oscillator strength indicates that this transition is forbid- den. The next transition occurs at $2.02 \mathrm{eV}$ with $f=5.6$ $\times 10^{-4}$ and confirms the $2.0 \mathrm{eV} \mathrm{OA}$ band with $f=10^{-4}$ to be due to the NBO center. This band has been assigned by Skuja $^{25}$ to a transition from the nonbonding orbitals of the oxygens in the vicinity of the terminal oxygen to the O- $2 p_{y}$ orbital localized on the latter atom. However, our calculations indicate that the excited state populated in this OA has $\sigma$-bonding character and is highly localized on the terminal oxygen. A Mulliken population analysis (MPA) of the singly occupied orbital in the $b^{2} A^{\prime}$ excited state shows a 77\% contribution of the $\mathrm{O}-2 p_{z}$ atomic orbital and a $13 \% \mathrm{Si}$ character, while the remainder arise from the three $\mathrm{OH}$ groups. Further evidence for this new assignment has been obtained considering a smaller cluster model without oxygens in the vicinity, i.e., from a $\mathrm{H}_{3} \mathrm{SiO}$. cluster model. After optimizing the geometry at the B3LYP/aug-cc-pVDZ level, we have calculated CASPT2 transition energies in a similar way as done for the larger cluster. Again we find the $a^{2} A^{\prime \prime}$ and $a^{2} A^{\prime}$ states to be almost degenerate. The transition to the $b^{2} A^{\prime}$ state occurs at $2.1 \mathrm{eV}$ and corresponds to a transition from the $\sigma$-bonding orbital with mainly $\mathrm{O}-2 p_{z}$ character, as in the $(\mathrm{OH})_{3} \mathrm{Si}-\mathrm{O}$. cluster. Hence, the oxygen atoms of the $\mathrm{OH}$ groups are not necessary to describe the transition observed at $2 \mathrm{eV}$.

There is experimental evidence that the $\mathrm{OA}$ at $2 \mathrm{eV}$ gives rise to a luminescence band at $1.9 \mathrm{eV}$. The calculation of emission energies is a very difficult task due to convergence problems when one tries to optimize the geometry for the excited state, usually a high root of a given symmetry. In an attempt to confirm such an assignment we have taken the following strategy. First, we assume that the only geometrical parameter susceptible to change from the $a^{2} A^{\prime \prime}$ ground state to the $b^{2} A^{\prime}$ excited state is the $\mathrm{Si}-\mathrm{O}$ distance. This is supported by the fact that the $\mathrm{OA}$ band at $2 \mathrm{eV}$ is well reproduced both with the $(\mathrm{OH})_{3} \mathrm{Si}-\mathrm{O}$. and the $\mathrm{H}_{3} \mathrm{Si}-\mathrm{O}$. cluster model. Using the same active space and cluster model as in Table II, we compute CASPT2 total energies of the $b^{2} A^{\prime}$ excited state at various geometries, varying only the $\mathrm{Si}-\mathrm{O}$ distance, while all other geometrical parameters are kept fixed. From this potential energy curve we extract the optimum Si-O distance for the $b^{2} A^{\prime}$ excited state, i.e., $1.704 \AA$, which turned out to be rather close to that corresponding to the ground state computed using the UMP2 method, i.e., $1.712 \AA$ (the CASPT2 equilibrium distance for the ground state is 1.714 , almost identical to the UMP2 value). At this new geometry, the luminescence band associated to the $b^{2} A^{\prime}$ state is computed at $1.97 \mathrm{eV}$ with a Stokes shift of 0.05 $\mathrm{eV}$. Experimentally, the luminescence is found only $0.1 \mathrm{eV}$ lower than the OA band. This gives additional theoretical proof that the NBO center is the origin of these two features.

Concerning the $4.8 \mathrm{eV}$ band, our CASPT2 results indicate that this transition arises from a $a^{2} A^{\prime \prime} \rightarrow c^{2} A^{\prime}$ transition, which comes out at $5.0 \mathrm{eV}$ with $f=4 \times 10^{-2}$. The relatively large oscillator strength is in agreement with the fact that this peak is the most intense one in the OA spectrum related to the NBO defect. The MPA of the open shell orbital of the excited state shows that the transition arises from the transfer of an electron out of the $2 p$ orbitals of the oxygens around the defect to the nonbridging oxygen. This is in contrast to 
TABLE III. CASPT2 excitation energies (in $\mathrm{eV}$ ) and oscillator strengths $f$ of the $(\mathrm{OH})_{3} \mathrm{SiOO}$. radical to model the peroxy oxygen radical defect in $\mathrm{SiO}_{2}$. The active space contains 12 orbitals and 11 electrons. CASPT2 leaves the core electrons ( $\mathrm{Si}-1 s, 2 s, 2 p$, and $\mathrm{O}-1 s$ ) frozen.

\begin{tabular}{ccc}
\hline \hline State & CASPT2 & $f$ \\
\hline$a^{2} A^{\prime \prime}$ & 0.00 & - \\
$a^{2} A^{\prime}$ & 0.71 & $1.9 \times 10^{-5}$ \\
$b^{2} A^{\prime \prime}$ & 5.49 & $1.4 \times 10^{-1}$ \\
$b^{2} A^{\prime}$ & 6.39 & $1.3 \times 10^{-5}$ \\
$c^{2} A^{\prime}$ & 6.83 & $9.1 \times 10^{-4}$ \\
$c^{2} A^{\prime \prime}$ & 7.59 & $2.7 \times 10^{-4}$ \\
\hline \hline
\end{tabular}

the interpretation of Skuja, ${ }^{25}$ who assigned the two experimental bands just opposite to our interpretation.

From our calculations another transition with appreciable intensity, comparable to that of the $4.8 \mathrm{eV}$ transition, is found at $4.08 \mathrm{eV}$; see Table II. This transition also arises from an excitation of an electron out of the $2 p$ orbitals of the oxygens of the three $\mathrm{OH}$ groups around the defect to the nonbonding oxygen. In the measurements there is no trace of a band in this region which can be correlated to this excitation. In fact, a band at $3.8 \mathrm{eV}$ originally assigned to a peroxy bridge ${ }^{58} \equiv \mathrm{Si}-\mathrm{O}-\mathrm{O}-\mathrm{Si} \equiv$, is most likely due to interstitial $\mathrm{Cl}_{2}{ }^{2}$ It is possible, however, that the transition computed at $4.08 \mathrm{eV}$ has a lower intensity than predicted by the calculations and that its presence is covered by the tails of the band at $4.8 \mathrm{eV}$.

\section{THE POR CENTER}

CASPT2 excitation energies and oscillator strengths for the transitions coming from the $a^{2} A^{\prime \prime}$ ground state to the lowest excited states have been computed for the POR center and summarized in Table III. In analogy to the NBO center, this defect site has been studied using the $(\mathrm{OH})_{3} \mathrm{Si}-\mathrm{OO}$. cluster model depicted in Fig. 2. The figure shows the most relevant distances and angles obtained from a B3LYP optimization of the ground state geometry. The spectroscopic parameters listed in Table III correspond to this geometry. The reference CASSCF wave function has been constructed from an active space with 12 orbitals and 11 electrons. The results are stable within $0.1 \mathrm{eV}$ to changes in the size of the basis set, the size of the active space (once a reasonable size has been reached, see Sec. III), and the level of theory used for the optimization of the geometry.

From Table III several conclusions can be drawn. First, we note that only the excitation at $5.5 \mathrm{eV}$ carries intensity; all other transitions show a much lower intensity. Second, no excitation is found below the intense one at $5.5 \mathrm{eV}$ besides the lowest transition at $0.7 \mathrm{eV}$. The character of the $0.7 \mathrm{eV}$ transition is reminiscent of that found for the HOO and $\mathrm{CH}_{3} \mathrm{OO} \cdot$ radicals, i.e., it corresponds to an internal transition on the terminal oxygen. This interpretation is in agreement with a previous UMP2 calculation by Radzig et al. ${ }^{35}$ Furthermore, the $5.5 \mathrm{eV}$ transition is assigned to an $a^{2} A^{\prime \prime} \rightarrow b^{2} A^{\prime \prime}$ excitation which basically arises from the transfer of an electron from the $\mathrm{O}$ directly connected to the $\mathrm{Si}$ atom $\left(\mathrm{O}_{1}\right)$ to the terminal oxygen $\left(\mathrm{O}_{2}\right)$. The higher excited states also correspond to internal transitions involving these two oxygens.
TABLE IV. Summary of proposed assignments of measured OA bands tentatively attributed to POR centers and comparison with computed bands at the CASPT2 level.

\begin{tabular}{|c|c|c|c|}
\hline \multicolumn{2}{|c|}{ Experimental assignment } & \multicolumn{2}{|c|}{ Theoretical assignment } \\
\hline$T_{e}$ & $f$ & $T_{e}$ & $f$ \\
\hline \multicolumn{2}{|c|}{ not observed } & 0.71 & $1.9 \times 10^{-5}$ \\
\hline 1.97 (Ref. 33) & $5.7 \times 10^{-4}($ Ref.33 $)$ & \multicolumn{2}{|c|}{ not supported } \\
\hline 4.8 (Ref. 29) & - & \multicolumn{2}{|c|}{ not supported } \\
\hline$\approx 5.4($ Ref. 31$)$ & $6 \times 10^{-2}($ Ref. 31$)$ & 5.49 & $1.4 \times 10^{-1}$ \\
\hline 7.6 (Ref. 16) & $6.5 \times 10^{-1}$ (Ref. 16) & \multicolumn{2}{|c|}{ not supported } \\
\hline
\end{tabular}

However, the fact that these excitations are predicted to carry very little intensity makes them less interesting and not easily observable experimentally. The CASPT2 results are in qualitative agreement with the previous theoretical calculations by Pacchioni and Ieranò, ${ }^{5}$ who also found the first transition at very low energy and the second one around $6.5 \mathrm{eV}$. Although these values are affected by the small basis sets and the limited treatment of the electron correlation, they reinforce the conclusion that there are no OA bands between $1 \mathrm{eV}$ and $5.4 \mathrm{eV}$ that can be associated with the POR center.

As stated in the Introduction, several bands has been assigned to the POR defect, but there seems to be no general agreement about these assignments. The fact that the CASPT2 excitation energies and oscillator strengths closely resemble the experimental data for three related molecules (two radicals, $\mathrm{HOO}$ - and $\mathrm{CH} 3 \mathrm{OO} \cdot$, and the $\mathrm{NBO}$ defect), for which the OA spectrum is unambiguously assigned, allows us to clarify the spectrum of the POR center. In Table IV we compare the different assignments proposed in the literature with our theoretical predictions. It is immediately clear that the only absorption band supported by our calculations is the one around $5.4 \mathrm{eV},{ }^{31}$ both the excitation energy and the oscillator strength coincide within the accuracy of the CASPT2 method. None of the other transitions can be confirmed. We do not find any transition either around $2 \mathrm{eV}$ or around 4.8 $\mathrm{eV}$, giving evidence that these $\mathrm{OA}$ bands do not arise from the POR center and are probably due to the NBO center described before. In the higher energy region we do find some transitions, but these carry much less intensity than the $7.6 \mathrm{eV}$ band observed by Friebele and co-workers and Stapelbroek et al. ${ }^{16,18}$ This band most likely originates from the presence of an oxygen vacancy which is known to give rise to a transition in this region. ${ }^{4}$

\section{CONCLUSIONS}

The nature of the transitions in a peroxy radical defect center, $\equiv \mathrm{Si}-\mathrm{O}-\mathrm{O}^{\circ}$, one of the most fundamental radiation induced defects in amorphous silicon dioxide, has been the subject of intense debate over the last three decades. ${ }^{16,18,23,28,30,32,33}$ So far, theoretical calculations have not been able to completely solve the problem. ${ }^{5}$ In this paper we have reported the results of high quality ab initio calculations with explicit inclusion of correlation effects. The CASSCF/CASPT2 method, especially designed with the aim of accurately reproducing excited state energies, has been applied to the study of cluster models of the POR center. The 
validity of this approach has been carefully tested by computing the excited state properties of two gas-phase molecular analogs of the $\mathrm{POR}$, the $\mathrm{HOO} \cdot$ and $\mathrm{CH}_{3} \mathrm{OO} \cdot$ radicals, and of another paramagnetic center in $\mathrm{SiO}_{2}$, i.e., the $\mathrm{NBO}$ center, $\equiv \mathrm{Si}-\mathrm{O}^{\circ}$. The results of the test calculations provide a direct validation of the approach used; the estimated error in the calculated transition energies does not exceed $0.2 \mathrm{eV}$. The main reason for the high accuracy in the calculated excitation energies despite the use of small clusters is the high degree of localization of the energy levels associated with the point defect.

The POR center has a first transition with very low intensity at $0.71 \mathrm{eV}$, followed by a second intense transition at $5.49 \mathrm{eV}$. No other transitions have been found below $6 \mathrm{eV}$. This theoretical result is consistent with the observation of a band with a maximum around $5.4 \mathrm{eV}$ observed by Radzig at the surface of mechanically activated silica, ${ }^{23,31}$ also, the oscillator strength of this band corresponds to that obtained from the CASPT2 calculations. On the basis of these results we can rule out that the POR defect gives rise to transitions in the regions of $2,4.8$, or $7.6 \mathrm{eV}$ as proposed in the literature. Finally, it is interesting to note that the allowed transition in the POR center at $5.4-5.5 \mathrm{eV}$ is very close to that predicted for the lowest singlet-singlet transition in a peroxy bridge center, $\equiv \mathrm{Si}-\mathrm{O}-\mathrm{O}-\mathrm{Si} \equiv$, which, according to coupled cluster calculations, occurs at $5.5 \mathrm{eV} .^{11}$

\section{ACKNOWLEDGMENTS}

The work has been financed by the Spanish 'Ministerio de Educación y Cultura' under CICyt Project No. PB 981216-C02-01 and 'Acción Integrada Hispano-Italiana, HI1998-0042'.

${ }^{1}$ Defects in $\mathrm{SiO}_{2}$ and Related Dielectrics: Science and Technology, edited by G. Pacchioni, L. Skuja, and D. Griscom, NATO ASI Series (Kluwer, Dordrecth, 2000).

${ }^{2}$ L. Skuja, J. Non-Cryst. Solids 239, 16 (1998).

${ }^{3}$ G. Pacchioni and G. Ieranò, Phys. Rev. B 56, 7304 (1997)

${ }^{4}$ G. Pacchioni and G. Ieranò, Phys. Rev. Lett. 79, 753 (1997)

${ }^{5}$ G. Pacchioni and G. Ieranò, Phys. Rev. B 57, 818 (1998).

${ }^{6}$ G. Pacchioni, G. Ieranò, and A. M. Márquez, Phys. Rev. Lett. 81, 377 (1998).

${ }^{7}$ G. Pacchioni and R. Ferrario, Phys. Rev. B 58, 6090 (1998).

${ }^{8}$ G. Pacchioni and A. Basile, Phys. Rev. B 60, 9990 (1999).

${ }^{9}$ B. B. Stefanov and K. Raghavachari, Appl. Phys. Lett. 71, 770 (1997).

${ }^{10}$ B. L. Zhang and K. Raghavachari, Phys. Rev. B 55, R15993 (1997).

${ }^{11}$ B. B. Stefanov and K. Raghavachari, J. Chem. Phys. 111, 8039 (1999).

${ }^{12}$ M. Boero, A. Pasquarello, J. Sarnthein, and R. Car, Phys. Rev. Lett. 78, 887 (1997).

${ }^{13}$ A. H. Edwards, J. Non-Cryst. Solids 187, 232 (1995).

${ }^{14}$ T. Uchino, Y. Kitagawa, and T. Yoko, J. Chem. Phys. 61, 234 (2000).

${ }^{15}$ G. Pacchioni, in Defects in $\mathrm{SiO}_{2}$ and Related Dielectrics: Science and Technology, edited by G. Pacchioni, L. Skuja, and D. Griscom, NATO ASI Series (Kluwer, Dordrecht, 2000).

${ }^{16}$ E. J. Friebele, D. L. Griscom, M. Stapelbroek, and R. A. Weeks, Phys. Rev. Lett. 42, 1346 (1979).
${ }^{17}$ R. A. Weeks, J. Appl. Phys. 27, 1376 (1956).

${ }^{18}$ M. Stapelbroek, D. L. Griscom, E. J. Friebele, and G. H. Sigel, J. NonCryst. Solids 32, 313 (1979).

${ }^{19}$ D. L. Griscom, J. Non-Cryst. Solids 149, 137 (1992).

${ }^{20}$ H. Nishikawa, R. Nakamura, Y. Ohki, and H. Hama, Phys. Rev. B 48, 15584 (1993).

${ }^{21}$ A. H. Edwards and W. B. Fowler, Phys. Rev. B 26, 6649 (1982).

${ }^{22}$ V. A. Radzig and A. V. Bystrikov, Kinet. Katal. 19, 713 (1978).

${ }^{23}$ V. A. Radzig, Chem. Phys. Reports 14, 1206 (1995).

${ }^{24}$ D. L. Griscom, J. Ceram. Soc. Jpn. 99, 923 (1991).

${ }^{25}$ L. Skuja, J. Non-Cryst. Solids 179, 51 (1994).

${ }^{26}$ Y. Sakurai, J. Appl. Phys. 87, 755 (2000).

${ }^{27}$ L. Skuja and A. R. Silin, Phys. Status Solidi A 56, K11 (1979).

${ }^{28}$ L. Skuja, K. Tanimura, and N. Itoh, J. Appl. Phys. 80, 3518 (1996).

${ }^{29}$ H. Hosono and R. A. Weeks, J. Non-Cryst. Solids 116, 289 (1990).

${ }^{30}$ R. A. Weeks, R. H. Magruder, and P. W. Wang, J. Non-Cryst. Solids 149, 122 (1992).

${ }^{31}$ A. A. Bobyshev and V. A. Radzig, Fiz. Khim. Stekla 14, 501 (1988).

${ }^{32}$ Y. Morimoto, R. A. Weeks, R. A. Magruder, and R. A. Zuhr, J. NonCryst. Solids 203, 55 (1996).

${ }^{33}$ D. L. Griscom and M. Mizuguchi, J. Non-Cryst. Solids 239, 66 (1998).

${ }^{34}$ E. O'Reilly and J. Robertson, Phys. Rev. B 27, 3780 (1983).

${ }^{35} \mathrm{~V}$. A. Radzig, A. A. Bobyshev, and I. N. Senchenia, in Thesis of VII Symposium on Optical and Spectral Properties of Glasses (Vavilov State Optical Institute, Leningrad, 1989), p. 258.

${ }^{36}$ K. Raghavachari and G. Pacchioni, J. Chem. Phys. (in press).

${ }^{37}$ D. E. Woon and T. H. Dunning, Jr., J. Chem. Phys. 98, 1358 (1993).

${ }^{38}$ K. Andersson, P.-A. Malmqvist, B. O. Roos, A. J. Sadlej, and K. Wolinski, J. Phys. Chem. 94, 5483 (1990).

${ }^{39}$ K. Andersson, P.-A. Malmqvist, and B. O. Roos, J. Chem. Phys. 96, 1218 (1992).

${ }^{40}$ B. O. Roos, K. Andersson, M. P. Fülscher, P.-A․ Malmqvist, L. SerranoAndrés, K. Pierloot, and M. Merchán, Adv. Chem. Phys. 93, 219 (1996).

${ }^{41}$ B. O. Roos, K. Andersson, M. P. Fülscher, L. Serrano-Andrés, K. Pierloot, M. Merchán, and V. Molina, J. Mol. Struct.: THEOCHEM 388, 257 (1996).

${ }^{42}$ A. Bernhardsson, N. Forsberg, P.-A․ Malmqvist, and B. O. Roos, J. Chem. Phys. 112, 2798 (2000).

${ }^{43}$ V. Molina, M. Merchán, B. O. Roos, and P.-Å. Malmqvist, Phys. Chem. Chem. Phys. 2, 2211 (2000).

${ }^{44}$ C. de Graaf, R. Broer, and W. C. Nieuwpoort, Chem. Phys. 208, 35 (1996).

${ }^{45}$ C. de Graaf and R. Broer, Phys. Rev. B 62, 702 (2000).

${ }^{46}$ C. Sousa, C. de Graaf, F. Illas, M. T. Barriuso, J. A. Aramburu, and M. Moreno, Phys. Rev. B 62, 13366 (2000).

${ }^{47}$ P.-Å. Malmqvist and B. O. Roos, Chem. Phys. Lett. 155, 189 (1989).

${ }^{48}$ M. J. Frisch et al., GAussian 98, Revision A.6 (Gaussian, Inc., Pittsburgh, PA, 1998).

${ }^{49}$ K. Andersson, M. R. A. Blomberg, M. P. Fülscher, G. Karlström, R. Lindh, P.-A. Malmqvist, P. Neogrády, J. Olsen, B. O. Roos, A. J. Sadlej, M. Schütz, L. Seijo, L. Serrano-Andrés, P. E. M. Siegbahn, and P.-O. Widmark, MOLCAS version 4 (University of Lund, Sweden, 1997).

${ }^{50}$ T. T. Paukert and H. S. Johnston, J. Chem. Phys. 56, 2824 (1972).

${ }^{51}$ C. J. Hochanadel, J. A. Ghormley, and P. J. Ogren, J. Chem. Phys. 56, 4426 (1972).

${ }^{52}$ S.-K. Shih and S. D. Peyerimhoff, Chem. Phys. 28, 299 (1978).

${ }^{53}$ F.-G. Simon, W. Schneider, and G. K. Moortgat, Int. J. Chem. Kinet. 22, 791 (1990).

${ }^{54}$ A. Fahr, A. H. Laufer, M. Krauss, and R. Osman, J. Phys. Chem. A 101, 4879 (1997)

${ }^{55}$ H. E. Hunziker and H. R. Wendt, J. Chem. Phys. 64, 3488 (1976).

${ }^{56}$ J. A. Jafri and D. H. Phillips, J. Am. Chem. Soc. 112, 2586 (1990).

${ }^{57}$ P.-O. Widmark, P.-A. Malmqvist, and B. O. Roos, Theor. Chim. Acta 77, 291 (1990).

${ }^{58}$ K. Awazu and H. Kawazoe, J. Non-Cryst. Solids 179, 215 (1994). 